\title{
The Effect of a Decrease in Intraocular Pressure on Optic Nerve Function in Patients with Optic Nerve Drusen
}

\author{
Dorota Pojda-Wilczek $^{a, b}$ Patrycja Wycisło-Gawron ${ }^{b}$ \\ ${ }^{a}$ Department of Ophthalmology, School of Medicine in Katowice, Medical University of Silesia, and \\ ${ }^{b}$ Ophthalmology Division, Kornel Gibiński University Clinic Centre of Medical University of Silesia in Katowice, \\ Katowice, Poland
}

\section{Keywords}

Pattern electroretinography · Visual-evoked potentials .

Neuroprotection · Optic nerve drusen · Visual field

\begin{abstract}
Purpose: The aim of the study was to compare optic nerve function in eyes with brinzolamide-reduced intraocular pressure (IOP) and the fellow eyes of patients with optic disk drusen (ODD). Methods: The study comprised 34 patients with bilateral ODD but no signs of any other ocular disease. The eyes with more advanced optic neuropathy were selected for treatment with an IOP-lowering drug, carbonic anhydrase inhibitor (brinzolamide); the fellow eyes served as the control. Static perimetry, pattern electroretinography (PERG), pattern visual-evoked potentials (PVEP), and retinal nerve fiber layer (RNFL) thickness were analyzed. The observation period was 12 months. Results: The eyes with brinzolamide-reduced IOP exhibited a statistically significant decrease in the mean defect index of static visual field ( $p=$ 0.03), an increase in PERG N95 amplitude (from 2.94 to $4.41 \mu \mathrm{V} ; p=0.0047$ ), and RNFL thickness stabilization. A statistically significant decrease in RNFL thickness (from 83.21 to $79.85 \mu \mathrm{m} ; p=0.0017$ ) was found in the control eyes. Conclusions: A decrease in IOP in eyes with ODD results in im-
\end{abstract}

\begin{tabular}{ll}
\hline KARGER & $\begin{array}{l}\text { ( } 2017 \text { The Author(s) } \\
\text { Published by S. Karger AG, Basel }\end{array}$ \\
E-Mail karger@karger.com & Karger \\
www.karger.com/ore & $\begin{array}{l}\text { This article is licensed under the Creative Commons Attribution- } \\
\text { NonCommercial-NoDerivatives 4.0 International License (CC BY- } \\
\text { NC-ND) (http://www.karger.com/Services/OpenAccessLicense) } \\
\text { Usage and distribution for commercial purposes as well as any dis- } \\
\text { tribution of modified material requires written permission. }\end{array}$
\end{tabular}

provement of retinal ganglion cell function and delays the progression of optic neuropathy. PERG should be performed in patients with ODD as it is a sensitive test for monitoring optic neuropathy.

(c) 2017 The Author(s)

Published by S. Karger AG, Basel

\section{Introduction}

Optic disk drusen (ODD) are congenital calcifications of the optic disk located anterior to the lamina cribrosa. The first report of ODD was a histologic description by Müller [1] in 1858, i.e., 7 years before the invention of an ophthalmoscope. He described crystalline granules (5$1,000 \mu \mathrm{m}$ in size) in the optic nerve head.

Clinical examination reveals ODD in 0.3 and $0.4 \%$ of adults and children, respectively whereas histological studies have reported a prevalence range of $0.5-3.7 \%$ [2, $3]$. This discrepancy is associated with the fact that only $40 \%$ of drusen are located on the surface of the optic nerve head and can be identified by a routine ophthalmoscopic examination as bright irregular deposits. Buried drusen may cause optic disk elevation, resulting in pseudopapilledema. About $69-91 \%$ of ODD are bilateral, and a female predilection (61-71\%) has been re- 
ported $[4,5]$. ODD occur most frequently in Caucasians [6].

ODD can be inherited with an irregularly autosomal dominant or autosomal recessive pattern [5]. Congenital risk factors for the development of drusen include inherited dysplasia of the optic disk and its blood supply, small optic disk size, and a small scleral canal [7].

Axonal degeneration is important in the pathogenesis of ODD. Abnormal axonal metabolism leads to intracellular mitochondrial calcification, axonal interruption, and calcium accumulation [5]. The small scleral canal causes optic nerve fiber compression and retinal ganglion cell (RGC) degeneration. Mechanical compression of prelaminar nerve fibers caused by drusen, impaired axonal transport, and ischemia within the optic nerve head result in the abnormal function of RGCs [5]. Optic neuropathy may be detected on visual-field and electrophysiological examinations and also when measuring retinal nerve fiber layer (RNFL) thickness. The frequency of visual-field defects in adults with ODD has been reported to range from 24 to $87 \%[8,9]$. The types of visual-field defects include arcuate defects, enlargement of the blind spot, and concentric contraction [6]. Schargus and Gramer [10] observed progressive visual-field deterioration with increasing age. Apart from the visual-field examination, electrophysiological examination is valuable in assessing optic neuropathy. The most common (79\% of the study eyes) ODD-associated abnormality on pattern electroretinogram (PERG) was a reduction in the amplitude of the N95 component; the P50 amplitude was reduced in only $17 \%$ of the eyes [11]. Compression of nerve fibers leads to a decrease of the P100 amplitude and an increase in P100 latency of pattern visual-evoked potentials (PVEP). An association was also found between PVEP abnormalities and the degree of RNFL thickness reduction [12]. ODD are usually associated with peripapillary RNFL thinning, which, in turn, is correlated with visual-field deterioration. Eyes with ODD are predisposed to various types of vascular complications including ischemic optic neuropathy, central and branch retinal vein occlusion, central and branch retinal artery occlusion, choroidal neovascular membrane, and retinal hemorrhage [5]. Ischemic optic neuropathy is the most common cause of sudden visual loss in patients with ODD due to an acute drop in optic nerve perfusion [2]. The improvement of optic nerve perfusion by lowering the intraocular pressure (IOP) could probably slow down the progression of optic neuropathy. The aim of this study was to compare optic nerve function in eyes with brinzolamide-reduced IOP and the fellow eyes of patients with ODD.

\section{Materials and Methods}

The study population comprised 34 patients (21 women and 13 men) diagnosed with bilateral ODD but with no signs of any other ocular disease. The patients were aged 18-68 years (mean: 42 years). Informed consent was obtained from each individual participant included in the study. The inclusion criteria were as follows: age $\geq 18$ years, willingness to participate in the study, and bilateral ODD. The exclusion criteria included treatment with antiglaucomatous agents, epilepsy or another central nervous system disease, nystagmus, visual acuity that did not allow fixation, photophobia, pregnancy, severe renal function impairment and/or liver function impairment, hypersensitivity to brinzolamide, a baseline IOP of $\leq 10 \mathrm{~mm} \mathrm{Hg}$, and other ocular diseases. The eyes with more advanced optic neuropathy (more advanced visual-field defects) were selected for treatment with an IOP-lowering drug (a carbonic anhydrase inhibitor), brinzolamide (the study group), and the fellow eyes served as the control. Brinzolamide was chosen because of its neuroprotective effect. All participants received the same brinzolamide (Azopt, Alcon). The study eyes were treated with 1 drop of brinzolamide twice daily. The patients were observed for a period of 12 months. Best-corrected visual acuity (BCVA), IOP measurement, static perimetry, PERG, a transient PVEP test, optical coherence tomography (OCT) to measure RNFL and central corneal thickness (CCT), a contrast sensitivity test, color fundus photography, and a fundus autofluorescence test were all performed at the baseline appointment. BCVA, IOP measurement, and static perimetry were repeated every 3 months. Electrophysiological examinations and the contrast sensitivity test were repeated every 6 months. The rest of the exams were repeated 1 year after baseline measurements. Static perimetry was performed using OCTOPUS 1-2-3, glaucomatous program tG1 (INTERZEAG, Switzerland). All the patients were familiar with visual-field examination. They had been examined at least twice a year for 3-5 years in our outpatient clinic. They knew the method and the perimeter very well. Only the most cooperative patients were chosen for this study. The analysis was performed with a reliability factor rating of $<15 \%$. IOP was measured using a Goldmann applanation tonometer on a slit-lamp. CCT was measured using OCT Visante (Zeiss, Germany). Electrophysiological examinations were performed using Reti-Port (Roland Consult, Germany). All examinations were compliant with International Society for Clinical Electrophysiology of Vision (ISCEV) standards [13, 14]. PVEP were obtained by pattern reversal stimulations of $1^{\circ}$ and $15^{\prime}$. The amplitude and latency of the major PVEP component (P100 wave) and amplitude and implicit time of the PERG P50 and N95 components were measured. PERG was recorded with a silver thread (DTL) electrode. RNFL thickness was determined with Cirrus HD-OCT (Carl Zeiss, Germany) after dilatation by $1 \%$ tropicamidum. Optic Disc Cube $200 \times 200$ protocol was selected for the study of the RNFL. Scans were accepted when they were complete, evenly exposed, the retina and RNFL border were properly seen, the recorded signal strength was $\geq 7$. An RNFL thickness analysis protocol was selected. The mean total RNFL thickness was analyzed. Color fundus photos and fundus autofluorescence imaging were performed using a fundus camera (TRC-NW7SF, Topcon, Japan). Contrast sensitivity was tested on the sine-wave contrast sensitivity test charts (Stereo Optical Co. Inc. Chicago, IL, USA).

To compare initial and follow-up visit data in the control and study groups, statistical analysis was performed using the paired- 
Table 1. Mean IOP, pachymetry, mean RNFL thickness, and median MD and LV indices of static perimetry in the study and control groups

\begin{tabular}{lll}
\hline & Study group & Control group \\
\hline IOP, mm Hg & & \\
$\quad$ At baseline & $17.03 \pm 1.97$ & $16.5 \pm 2.01$ \\
At 12 -month follow-up & $11.09 \pm 1.83$ & $15.5 \pm 2.48$ \\
$p$ value & $<\mathbf{0 . 0 0 0 1}$ & $\mathbf{0 . 0 2 0 7}$ \\
Pachymetry, $\mu \mathrm{m}$ & & \\
At baseline & $550.97 \pm 22.45$ & $551.73 \pm 23.38$ \\
At 12-month follow-up & $555.91 \pm 23.57$ & $555.23 \pm 24.07$ \\
$p$ value & $\mathbf{0 . 0 0 0 3}$ & $\mathbf{0 . 0 4 8 7}$ \\
RNFL thickness, $\mu \mathrm{m}$ & & \\
At baseline & $75.5 \pm 18.97$ & $83.21 \pm 20.37$ \\
At 12 -month follow-up & $74.26 \pm 21.18$ & $79.85 \pm 21.41$ \\
$p$ value & 0.3 & $\mathbf{0 . 0 0 1 7}$ \\
Static perimetry, dB & & \\
At baseline (MD; LV) & $3.85 / 0.79 ; 9.25 / 4.2$ & $3.2 / 0.68 ; 7.15 / 5.09$ \\
At 12 -month follow-up (MD; LV) & $2.5 / 0.8 ; 8.45 / 4.0$ & $1.8 / 0.7 ; 6.4 / 4.73$ \\
$p$ value (MD; LV) & $\mathbf{0 . 0 3 7 8 ; 0 . 7 9 5 6}$ & $0.2058 ; 0.791$ \\
\hline
\end{tabular}

Values are expressed as mean \pm SD or median/SEM. To compare baseline and follow-up data, a paired-samples $t$ test was used for IOP, RNFL thickness, and CCT, and the Wilcoxon matched-pairs test was used for the MD and LV factors. Bold type denotes statistical significance. IOP, intraocular pressure; RNFL, retinal nerve fiber layer; $\mathrm{MD}$, mean defect; $\mathrm{LV}$, lost variance.

samples $t$ test for comparison of normal-distribution data (IOP, RNFL thickness, and CCT) and the Wilcoxon matched-pairs test for comparison of nonnormally distributed data, i.e. the mean defect (MD) and lost variance (LV) factors of static perimetry, and the parameters of PVEP and PERG. Statistical analysis for independent samples (study group vs. control group) of initial values was done using the Welch $t$ test (normal distribution) or the Mann-Whitney U test (nonnormal distribution). The normality of distribution in groups of data was checked by the Shapiro-Wilk test. The level of statistical significance was set at $p<0.05$.

The research was approved by Ethics Committee of Medical University of Silesia in Katowice (decision No. KNW/0022/ KB1/59/12).

\section{Results}

The mean BCVA (decimal) was 1.0 and 0.96 in the study and control eyes, respectively. The BCVA did not change in either group during the observation period. At baseline, both groups exhibited comparable IOP values. After 12 months of observation, IOP had decreased in study and control eyes by an average of $5.94 \mathrm{~mm} \mathrm{Hg}(p<$ $0.0001)$ and $1 \mathrm{~mm} \mathrm{Hg}(p=0.0207)$, respectively (Table 1$)$.

At baseline, the visual-field defects were more advanced in the study group than in controls, as demon- strated by the higher MD and LV indices; however, the differences were not statistically significant. After 12 months, a statistically significant decrease in the MD index was found in the study eyes, i.e., from 3.85 to 2.5 ( $p=$ 0.0378 ). There was no visual-field improvement in the control eyes; the MD decreased from 3.2 to 1.8 ( $p=$ $0.2058)$. The LV index showed statistically nonsignificant changes in both groups (Table 1); LV decreased from 9.25 to 8.45 in study eyes $(p=0.7956)$ and from 7.15 to 6.4 in control eyes $(p=0.791)$.

There was no statistically significant difference in CCT at baseline (a mean of $550.97 \mu \mathrm{m}$ in study eyes and 551.73 $\mu \mathrm{m}$ in control eyes). During the observation period, CCT increased significantly in the study eyes to $555.91 \mu \mathrm{m}$ $(p=0.0003)$; it also increased in the control eyes to 555.23 $\mu \mathrm{m}$, but the result was of borderline significance $(p=$ 0.0487; Table 1).

A statistically significant increase in the median N95 wave amplitude (AmplN95) was seen in the study eyes at 12 months after the treatment had been initiated, i.e., from 2.94 to $4.41 \mu \mathrm{V}(p=0.0047)$. The median P50 wave amplitude and median implicit times of P50 and N95 waves did not change significantly during the observation period in either group (Table 2). 
Table 2. Basic PERG parameters in the study and control groups

\begin{tabular}{|c|c|c|c|c|c|c|c|c|c|c|c|c|c|c|c|c|}
\hline \multirow[t]{2}{*}{ PERG } & \multicolumn{8}{|c|}{ Study group } & \multicolumn{8}{|c|}{ Control group } \\
\hline & median & SEM & median & SEM & median & SEM & median & SEM & median & SEM & median & SEM & median & SEM & median & SEM \\
\hline At baseline & 2.25 & 0.21 & 51.5 & 0.71 & 2.94 & 0.36 & 93.5 & 1.66 & 2.31 & 0.29 & 51 & 0.91 & 3.63 & 0.39 & 94.5 & 2.13 \\
\hline At 12 months & 2.36 & 0.28 & 50.4 & 0.62 & 4.41 & 0.4 & 92.8 & 1.26 & 2.68 & 0.27 & 50 & 0.71 & 4.26 & 0.34 & 93.85 & 1.28 \\
\hline$p$ value & \multicolumn{2}{|l|}{0.12} & \multicolumn{2}{|c|}{0.77} & \multicolumn{2}{|c|}{0.0047} & \multicolumn{2}{|c|}{0.22} & \multicolumn{2}{|l|}{0.26} & \multicolumn{2}{|c|}{0.53} & \multicolumn{2}{|c|}{0.496} & \multicolumn{2}{|c|}{0.97} \\
\hline
\end{tabular}

The Wilcoxon matched-pairs test was used for statistical analysis. Bold type denotes statistical significance. PERG, pattern electroretinography; ampl, amplitude.

Table 3. Basic PVEP parameters in the study and control groups

\begin{tabular}{|c|c|c|c|c|c|c|c|c|c|c|c|c|c|c|c|c|}
\hline \multirow[t]{3}{*}{ PVEP } & \multicolumn{8}{|c|}{ Study group } & \multicolumn{8}{|c|}{ Control group } \\
\hline & \multicolumn{2}{|c|}{$\begin{array}{l}\text { ampl P100 } \\
1^{\circ}, \mu \mathrm{V}\end{array}$} & \multicolumn{2}{|c|}{$\begin{array}{l}\text { ampl P100 } \\
15^{\prime}, \mu \mathrm{V}\end{array}$} & \multicolumn{2}{|l|}{$\begin{array}{l}\text { lat P100 } \\
1^{\circ}, \mathrm{ms}\end{array}$} & \multicolumn{2}{|l|}{$\begin{array}{l}\text { lat P100 } \\
15^{\prime}, \mathrm{ms}\end{array}$} & \multicolumn{2}{|c|}{$\begin{array}{l}\text { ampl P100 } \\
1^{\circ}, \mu \mathrm{V}\end{array}$} & \multicolumn{2}{|c|}{$\begin{array}{l}\text { ampl P100 } \\
15^{\prime}, \mu \mathrm{V}\end{array}$} & \multicolumn{2}{|c|}{$\begin{array}{l}\text { lat P100 } \\
1^{\circ}, \mathrm{ms}\end{array}$} & \multicolumn{2}{|l|}{$\begin{array}{l}\text { lat P100 } \\
15^{\prime}, \mathrm{ms}\end{array}$} \\
\hline & median & SEM & median & SEM & median & SEM & median & SEM & median & SEM & median & SEM & median & SEM & median & SEM \\
\hline At baseline & 8.38 & 0.77 & 9.64 & 1.33 & 105 & 1.3 & 117.5 & 1.71 & 7.7 & 0.84 & 11 & 1.29 & 104.5 & 1.11 & 113.5 & 2.64 \\
\hline \multirow{2}{*}{$\begin{array}{l}\text { At } 12 \text { months } \\
p \text { value }\end{array}$} & 8.27 & 0.81 & 9.87 & 1.21 & 105 & 1.18 & 114 & 1.54 & 9.82 & 0.86 & 9.92 & 1.2 & 105 & 1.13 & 112.55 & 1.98 \\
\hline & \multicolumn{2}{|c|}{0.73} & \multicolumn{2}{|c|}{0.3} & \multicolumn{2}{|c|}{0.95} & \multicolumn{2}{|c|}{0.71} & \multicolumn{2}{|l|}{0.2} & \multicolumn{2}{|l|}{0.97} & \multicolumn{2}{|c|}{0.11} & \multicolumn{2}{|c|}{0.96} \\
\hline
\end{tabular}

Wilcoxon matched pairs test was used for statistical analysis. PVEP, pattern visual-evoked potentials; ampl, amplitude; lat, latency; med, median.

In PVEP, the P100 wave amplitude and latency did not change significantly during the observation period (Table 3).

At baseline, the mean thickness of the RNFL was less in the study eyes than in the controls. By 12 months, RNFL thickness decreased had significantly in the control eyes, i.e., from 83.21 to $79.85 \mu \mathrm{m}(p=0.0017)$ while no significant changes were noted in the study eyes $(75.5 \mu \mathrm{m}$ initially and $74.26 \mu \mathrm{m}$ finally $(p=0.3$; Table 1$)$.

There were no significant differences in contrast sensitivity test during the observation period. The mean contrast values in treated eyes for the spatial frequencies 1.5, $3,6,12$, and 18 cycles per degree were, respectively, 42 , $74,53,11$, and 2 at baseline and 46, 80, 58, 15, and 3 after 12 months. In control eyes the corresponding values were $52,81,56,11$, and 3 at baseline and 48, 81, 62, 14, and 3 at the end of observation.

\section{Discussion}

ODD can cause progressive optic neuropathy. ODDrelated nerve fiber compression causes visual-field defects, alters the results of electrophysiological examina- tions, and reduces RNFL thickness. Mustonen [15] examined a large group of patients with ODD and described visual-field defects in $73 \%$ of the eyes with drusen. He observed visual-field deterioration in $22 \%$ of the patients, which confirms that ODD may underlie progressive optic neuropathy. Grippo et al. [16] reported that visual-field defects were more frequent in eyes with ODD and coexisting ocular hypertension. Spalding [17] recommended reducing the IOP in patients with concomitant occurrence of ocular hypertension and ODD. Schargus and Gramer [10] examined 16 patients with ODD who had undergone IOP-lowering therapy and 16 ODD patients without treatment. They observed a significantly slower progression of visual-field loss in the eyes with decreased IOP. In our study, visual-field defects were found in all patients, and these ranged from small relative scotomas to advanced visual-field loss. Defects had highly individual character and depended on the localization of ODD. The MD index of static perimetry was chosen to compare visual-field examinations before and after the treatment. We noted visual-field improvement as a statistically significant decrease in MD index in the treated eyes. In individual examinations, there was little improvement of sensitivity and decrease of area of scotomas. 
Other important indicators of improvement in optic nerve function in the brinzolamide-treated eyes were a nonsignificant change in RNFL thickness, but the control eyes exhibited a statistically significant reduction in RNFL thickness. Roh et al. [18] analyzed visual-field defects and RNFL thickness in the upper and lower quadrants in patients with bilateral ODD. He concluded that OCT was able to detect RNFL thinning in eyes with superficial ODD. The thinning revealed on OCT correlated with visual-field deficits and RNFL loss seen on red-free photography. Local RNFL thinning was noted in those quadrants in which drusen were located, and corresponded to the location of visual-field defects. Stevens and Newman [12] also found a relationship between the extent of visual-field loss and RNFL thinning.

The available literature provides no established effective treatment for ODD. Our study demonstrated that eyes treated with the IOP-lowering drug, brinzolamide, for 12 months, exhibited a delay in the progression of optic neuropathy. The most important indicator of this was a significantly higher amplitude of the PERG N95 component compared to baseline, which confirmed better functioning of the RGCs. No statistically significant changes were observed with respect to P50 amplitude. The P50 wave reflects the function of the RGCs and macular photoreceptors [19]. An improvement in N95 amplitude in the brinzolamide-treated eyes indicated that it was possible to partially restore the function of the RGCs in patients with ODD. PERG is a very sensitive test to detect the initial stages of optic neuropathy; it also allows the monitoring of changes in the RGC layer. Bach and Hoffman [20] concluded that PERG assists in identifying patients with elevated IOP in whom glaucoma damage is incipient before visual-field changes occur. Scholl et al. [11] reported that a reduction in the amplitude of the PERG N95 component was the most common abnormality seen in drusen-associated optic neuropathy (in $79 \%$ of cases). Reduced amplitude of the N95 wave reflects dysfunction of the RGCs located more peripherally to the macula. They observed that a low N95 amplitude was frequently associated with visual-field defects and impaired VEP. Stevens and Newman [12] found a VEP abnormality in $97.2 \%$ of the eyes with ODD. Grippo et al. [21] obtained different results. They did not observe a statistically significant delay in P100 latency following the check size of $1^{\circ}$ and $15^{\prime}$. The abovementioned studies compared eyes with and without ODD. In our study, all the eyes examined had ODD.

Our study did not reveal statistically significant changes in the amplitude and latency of the PVEP P100 wave. This could be attributed to the relatively short time of observation (12 months). PVEP have higher diagnostic accuracy in the long-term assessment of ODDassociated optic neuropathy when compared to optic nerve damage monitoring at a specific time point. PVEP are widely used for testing the function of the optic nerve and visual pathway but are not recommended for the early detection and monitoring of glaucoma. We had to compare our patients with glaucoma patients from other studies as we found no similar studies concerning ODD patients. Bach and Hoffman [20] explained that PVEP reflect the activity of later stages in the visual processing chain which are subject to gain control (possibly masking early changes) between RGC activity and cortical response.

Brinzolamide applied topically to 1 eye significantly lowered IOP in both eyes. The mean reduction was 5.4 and $1 \mathrm{~mm} \mathrm{Hg}$ in the treated and fellow eyes, respectively. This bilateral effect was associated with the systemic action of brinzolamide, which, although a topical agent, is absorbed into the bloodstream and accumulates in erythrocytes due to carbonic anhydrase binding.

Our study showed a statistically significant increase in CCT in the eyes after 12 months of brinzolamide treatment. However, the increase had no impact on the quality of vision. CCT in the fellow eyes also increased, which may confirm not only the topical but also the systemic effects of brinzolamide, although this needs further investigation.

Brinzolamide therapy should be considered in all patients with drusen-associated optic neuropathy. A decrease in IOP delays the progression of optic neuropathy. PERG is an objective and sensitive test that facilitates monitoring of optic neuropathy progression, not only in glaucoma but also in ODD. Our study had some limitations due to its small sample size and relatively short time of observation which might have contributed to the statistical results. We hope that this study will give rise to further long-lasting investigations.

\section{Conclusions}

A decrease in IOP in eyes with ODD results in an improvement in RGC function and delays the progression of optic neuropathy. PERG should be performed in patients with ODD as it is a sensitive test for optic neuropathy monitoring. 


\section{Acknowledgements}

The study was supported by the Medical University of Silesia, grant No. KNW-1-134/N/3/0.

\section{Disclosure Statement}

The authors declare no conflict of interests.

\section{References}

1 Friedman AH, Gartner S, Modi SS: Drusen of the optic disk. A retrospective study in cadaver eyes. Br J Ophthalmol 1975;59:413-421.

2 Davis PL, Jay WM: Optic nerve head drusen. Semin Ophthalmol 2003;18:222-242.

3 Wilkins JM, Pomeranz HD: Visual manifestations of visible and buried optic disk drusen. J Neuroophthalmol 2004;24:125-129.

4 Antcliff RJ, Spalton DJ: Are optic disk drusen inherited? Ophthalmology 1999;106:12781281.

5 Auw-Haedrich C, Staubach F, Witschel H: Optic disk drusen. Surv Ophthalmol 2002;47: 515-532.

6 Morris R, Ellerbrock J, Hamp A, Joy J, Roels $\mathrm{P}$, Davis C: Advanced visual field loss secondary to optic nerve head drusen: case report and literature review. Optometry 2009;80:83100 .

7 Mullie MA, Sanders MD: Scleral canal size and optic nerve head drusen. Am J Ophthalmol 1985;99:356-359.

8 Lee AG, Zimmerman MB: The rate of visual field loss in optic nerve head drusen. Am J Ophthalmol 2005;139:1062-1066.

9 Katz BJ, Pomeranz HD: Visual field defects and retinal nerve fiber layer defects in eyes with buried optic nerve drusen. Am J Ophthalmol 2006; 141:248-253.
10 Schargus M, Gramer E: Optic disc drusen. Ophthalmologe 2008;105:693-710.

11 Scholl GB, Song HS, Winkler DE, Wray SH The pattern visual evoked potential and pattern electroretinogram in drusen-associated optic neuropathy. Arch Ophthalmol 1992; 110:75-81.

12 Stevens RA, Newman NM: Abnormal visualevoked potentials from eyes with optic nerve head drusen. Am J Ophthalmol 1981;92:857862.

13 Odom JV, Bach M, Brigell M, Holder GE, McCulloch DL, Tormene AP: ISCEV standard for clinical visual evoked potentials (2009 update). Doc Ophthalmol 2010;120: 111-119.

14 Bach M, Brigell MG, Hawlina M, Holder GE, Johnson MA, McCulloch DL, Meigen T, Viswanathan S: ISCEV standard for clinical pattern electroretinography (PERG) - 2012 update. Doc Ophthalmol 2013;126:1-7.

15 Mustonen E: Pseudopapilloedema with and without verified optic disc drusen. A clinical analysis II: visual fields. Acta Ophthalmol 1983;61:1057-1066.
16 Grippo TM, Shihadeh WA, Schargus M, Gramer E, Tello C, Liebmann JM, Ritch R: Optic nerve head drusen and visual field loss in normotensive and hypertensive eyes. J Glaucoma 2008;17:100-104.

17 Spalding J: Visual-field loss with optic nerve drusen and ocular hypertension: a case report. Optometry 2002;73:24-32.

18 Roh S, Noecker RJ, Schuman JS, Hedges TR 3rd, Weiter JJ, Mattox C: Effect of optic nerve head drusen on nerve fiber layer thickness. Ophthalmology 1998;105:878-885.

19 Karaśkiewicz J, Lubiński W, Penkala K: Electrophysiological tests in evaluation of glaucoma and ocular hypertension treatment-up to date knowledge. A review. Klin Oczna 2013; 115:148-151.

20 Bach M, Hoffmann MB: Update on the pattern electroretinogram in glaucoma. Optom Vis Sci 2008;85:386-395.

21 Grippo TM, Ezon I, Kanadani FN, Wangsupadilok B, Tello C, Liebmann JM, Ritch R, Hood DC: The effects of optic disc drusen on the latency of the pattern-reversal checkerboard and multifocal visual evoked potentials. Invest Ophthalmol Vis Sci 2009;50: 4199-4204. 